\title{
On the Principle of Least Action
}

\author{
Vu B Ho* \\ Advanced Study, 9 Adela Court, Mulgrave, Victoria 3170, Australia \\ *Corresponding author: vubho@bigpond.net.au
}

\begin{abstract}
Investigations into the nature of the principle of least action have shown that there is an intrinsic relationship between geometrical and topological methods and the variational principle in classical mechanics. In this work, we follow and extend this kind of mathematical analysis into the domain of quantum mechanics. First, we show that the identification of the momentum of a quantum particle with the de Broglie wavelength in 2-dimensional space would lead to an interesting feature; namely the action principle $\delta S=0$ would be satisfied not only by the stationary path, corresponding to the classical motion, but also by any path. Thereupon the Bohr quantum condition possesses a topological character in the sense that the principal quantum number $n$ is identified with the winding number, which is used to represent the fundamental group of paths. We extend our discussions into 3-dimensional space and show that the charge of a particle also possesses a topological character and is quantised and classified by the homotopy group of closed surfaces. We then discuss the possibility to extend our discussions into spaces with higher dimensions and show that there exist physical quantities that can be quantised by the higher homotopy groups. Finally we note that if Einstein's field equations of general relativity are derived from Hilbert's action through the principle of least action then for the case of $n=2$ the field equations are satisfied by any metric if the energy-momentum tensor is identified with the metric tensor, similar to the case when the momentum of a particle is identified with the curvature of the particle's path.
\end{abstract}

Keywords: principle of least action, Feynman path integral method, classical mechanics, quantum mechanics, general relativity

Cite This Article: Vu B Ho, "On the Principle of Least Action." International Journal of Physics, vol. 6, no. 2 (2018): 47-52. doi: 10.12691/ijp-6-2-4.

\section{Introduction}

In classical physics, the principle of least action is a variational principle that can be used to determine uniquely the equations of motion for various physical systems. However, recently the principle of least action that is associated with new concepts of Lagrangian symmetry has been proposed and studied by many authors. It has also been shown that different formulations of the principle of least action can be constructed for a physical system that is described by a particular system of differential equations [1-5]. Furthermore, new approach to the theories of gravitation using the principle of least action has also been considered [6]. To extend these investigations, in this work we will consider the case in which the principle of least action can be applied to both classical physics and quantum physics.

In the old quantum theory, the Bohr quantum condition $\oint p d s=n h$, where $p$ is the momentum of a particle, $h$ is Planck constant and $n$ is a positive integer, played a crucial role in the quantum description of a physical system, although it had been introduced into the quantum theory in an ad hoc manner [7]. However, except for the quantum condition imposed on the orbital angular momentum, the Bohr model was based entirely on the classical dynamics of Newtonian physics. In this case, it seems natural to raise the question as to whether the Bohr quantum condition can also be described in a classical way. In classical mechanics, the actual path of a particle is found by extremising the action integral of the particle [8]. On the other hand, in quantum mechanics, the wave equation of a particle can be found by applying the Feynman path integral formulation which assumes the particle can take any trajectory [9]. The question then arises as to whether it is possible for the action integral to be extremised by any path at the quantum level. In this work we show that this problem may be investigated in terms of geometry and topology, and it transpires that topology may play an important role in the determination of the nature of a quantum observable $[10,11]$.

\section{Principle of Least Action in Classical Mechanics}

The investigation of the relationship between physics and geometry had been carried out and culminated with the development of the principle of least action. In 1744, Euler developed and published his work on this variational principle for the dynamics of a particle moving in a plane curve [12]. On the one hand, the dynamics of a particle can be studied by using the principle of least action. On the other hand, the path of a particle can also be determined by using geometrical methods, since a path can be constructed if the curvature is known at all points on the path. Euler showed the equivalence between these 
two methods by calculating the radius of curvature of the path directly and by means of the variational principle. Consider a particle moving in a plane under the influence of a force. Let $F_{x}$ and $F_{y}$ be the forces per unit mass in the $x$-direction and the $y$-direction, respectively. The normal acceleration $a_{n}$ of the particle is given by

$$
a_{n}=\left(F_{x} \frac{d y}{d x}-F_{y}\right)\left(1+\left(\frac{d y}{d x}\right)^{2}\right)^{-\frac{1}{2}} .
$$

If $\rho$ is the radius of curvature, then $a_{n}=-v^{2} / \rho$, where $v$ is the speed of the particle. This result can also be obtained by using the variational principle $\delta S=0$, where $S$ is defined by

$$
S=\int p d s=\int p \sqrt{1+\left(\frac{d y}{d x}\right)^{2}} d x
$$

The condition $\delta S=0$ leads to the stationary condition

$$
\frac{d}{d x}\left(\frac{\partial}{\partial \frac{d y}{d x}}\left(v \sqrt{1+\left(\frac{d y}{d x}\right)^{2}}\right)\right)-\frac{\partial}{\partial y}\left(v \sqrt{1+\left(\frac{d y}{d x}\right)^{2}}\right)=0 .
$$

Employing the relations $\partial v^{2} / \partial x=2 F_{x}$ and $\partial v^{2} / \partial y=$ $2 F_{y}$, the stationary condition given by Equation (3) then leads to $a_{n}=-v^{2} / \rho$. This result reveals an intrinsic relationship between geometrical methods and the variational principle in classical mechanics.

\section{Principle of Least Action in Quantum Mechanics}

We will now extend this geometrical analysis into the domain of quantum mechanics. We show that the identification of the momentum of a quantum particle with the de Broglie wavelength leads to an interesting feature; namely the action principle $\delta S=0$ is satisfied not only by the stationary path corresponding to the classical motion, but also by any path. In this case the Bohr quantum condition possesses a topological character in the sense that the principal quantum number $n$ is identified with the winding number, which is used to represent the fundamental group of paths [13]. Consider a curve in the three-dimensional spatial continuum $\mathrm{R}^{3}$ which is a topological image of an open segment of a straight line. The curve can be represented by a real vector function $\mathbf{r}(s)$. For the Bohr planar model, we utilise the fundamental homotopy group and it is convenient to consider curves that may have points that correspond to more than one value of the parameter $s$. However, whenever the single-valuedness of the representation $\mathbf{r}(s)$ is required for differential analysis of the motion, the uncertainty principle in quantum mechanics may be invoked to shift the self-intersecting points into the third dimension to make the curve single-valued for the whole domain of definition. This is a consequence of quantum mechanics which does not allow both the momentum and coordinate associated with the third dimension to vanish simultaneously [10]. In differential geometry, the position vector $\mathbf{r}(s)$, the unit tangent vector $\mathbf{t}(s)$, the unit principal normal vector $\mathbf{p}(s)$ and the unit binormal vector $\mathbf{b}(s)$, defined by the relation $\mathbf{b}(s)=\mathbf{t}(s) \times \mathbf{p}(s)$, satisfy the Frenet equations $[14,15]$

$$
\frac{d \mathbf{t}}{d s}=\kappa \mathbf{p}, \frac{d \mathbf{p}}{d s}=-\kappa \mathbf{t}+\varrho \mathbf{b}, \frac{d \mathbf{b}}{d s}=-\varrho \mathbf{p}
$$

where $\kappa(s)$ and $\varrho(s)$ are the curvature and the torsion respectively, and $d s=\sqrt{d \mathbf{r} \cdot d \mathbf{r}}$ is the linear element. If we consider the motion of a particle in a plane, as in the case of Bohr's model of a hydrogen-like atom, the Frenet equations reduce to

$$
\frac{d \mathbf{t}}{d s}=\kappa \mathbf{p}, \frac{d \mathbf{p}}{d s}=-\kappa \mathbf{t} .
$$

By differentiation, we obtain the following system of differential equations

$$
\begin{aligned}
& \frac{d^{2} \mathbf{t}}{d s^{2}}-\frac{d(\ln \kappa)}{d s} \frac{d \mathbf{t}}{d s}+\kappa^{2} \mathbf{t}=0 \\
& \frac{d^{2} \mathbf{p}}{d s^{2}}-\frac{d(\ln \kappa)}{d s} \frac{d \mathbf{p}}{d s}+\kappa^{2} \mathbf{p}=0 .
\end{aligned}
$$

If the curvature $\kappa(s)$ is assumed to vary slowly along the curve $\mathbf{r}(s)$, so that the condition $d(\ln \kappa) / d s=0$ can be imposed, then $\mathbf{t}(s)$ and $\mathbf{p}(s)$ may be regarded as being oscillating with a spatial period, or wavelength, $\lambda$, whose relationship to the curvature $\kappa$ is found as

$$
\kappa=\frac{2 \pi}{\lambda} \text {. }
$$

In the case of the Bohr's planar model of a hydrogenlike atom with circular orbits, the condition $d(\ln \kappa) / d s=$ 0 is always satisfied, since the curvature remains constant for each of the orbits. In order to incorporate this elementary differential geometry into quantum mechanics, we identify the wavelength defined in Equation (8) with the de Broglie's wavelength of a particle. This seems to be a natural identification since the spatial period $\lambda$ is the wavelength of the unit tangent vector $\mathbf{t}(s)$. With this assumption, the momentum $p$ of the particle and the curvature $\kappa$ are related through the relation

$$
p=\hbar \kappa \text {. }
$$

We now want to show how this result leads to Bohr's postulate of the quantisation of angular momentum. It should be mentioned here that this can only be discussed in terms of Bohr's quantum theory, or Feynman's path integral methods, since these formulations do require concepts employed in classical physics, especially the concept of classical paths of a particle [9]. According to the canonical formulation of classical physics, the particle dynamics is governed by the action principle $\delta S=$ $\delta \int p d s=0$. Using the relationship $p=\hbar \kappa$ given in Equation (9) and the expression for the curvature $\kappa$ of the path $f(x)$ of a particle in a plane, $\kappa=f^{\prime \prime} /\left(1+f^{\prime 2}\right)^{3 / 2}$, where $f^{\prime}=d f / d x$ and $f^{\prime \prime}=d^{2} f / d x^{2}$, the action integral $S$ takes the form

$$
S=\int \hbar \kappa d s=\int \frac{\hbar f^{\prime \prime}}{1+f^{\prime 2}} d x
$$


It is shown in the calculus of variations that to extremise the integral $S=\int L\left(f, f^{\prime}, f^{\prime \prime}, x\right) d x$, the function $f(x)$ must satisfy the differential equation [16]

$$
\frac{\partial L}{\partial f}-\frac{d}{d x} \frac{\partial L}{\partial f^{\prime}}+\frac{d^{2}}{d x^{2}} \frac{\partial L}{\partial f^{\prime \prime}}=0 .
$$

However, with the functional of the form given in Equation (10), $L=\hbar f^{\prime \prime} /\left(1+f^{\prime 2}\right)$, it is straightforward to verify that the differential equation given in Equation (11) is satisfied by any function $f(x)$. This result may be considered as a foundation for the Feynman's path integral formulation of quantum mechanics, which uses all classical trajectories of a particle in order to calculate the transition amplitude of a quantum mechanical system $[9,12]$. Since any path can be taken by a particle moving in a plane, if the orbits of the particle are closed, it is possible to represent each class of paths of the fundamental homotopy group of the particle by a circular path, since topologically, any path in the same equivalence class can be deformed continuously into a circular path [13]. This validates Bohr's assumption of circular motion for the electron in a hydrogen-like atom. This assumption then leads immediately to the Bohr quantum condition

$$
\oint p d s=\hbar \oint \kappa d s=\hbar \oint \frac{d s}{r}=\hbar \oint d \theta=n h .
$$

The Bohr quantum condition possesses a topological character in the sense that the principal quantum number $n$ is identified with the winding number which is used to represent the fundamental homotopy group of paths of the electron in the hydrogen atom.

It is interesting to note that our discussions for the dynamics of a particle in a three-dimensional spatial continuum can be extended to a three-dimensional temporal manifold. Mathematically, a temporal manifold can be considered as a three-dimensional Euclidean continuum whose radial time can be identified with the one-dimensional time in physics [17]. In this case we can also define the temporal position, or moment, vector $\mathbf{t}(\tau)$, the unit temporal tangent vector $\mathbf{t}_{T}(\tau)$, the unit temporal principal normal vector $\mathbf{p}(\tau)$ and the unit temporal binormal vector $\mathbf{b}(\tau)$, defined by the relation $\mathbf{b}(\tau)=$ $\mathbf{t}_{T}(\tau) \times \mathbf{p}(\tau)$. These mathematical objects satisfy the temporal Frenet equations

$$
\frac{d \mathbf{t}_{T}}{d \tau}=\kappa \mathbf{p}, \frac{d \mathbf{p}}{d \tau}=-\kappa \mathbf{t}_{T}+\varrho \mathbf{b}, \frac{d \mathbf{b}}{d \tau}=-\varrho \mathbf{p}
$$

where $\kappa(\tau)$ and $\varrho(\tau)$ are the temporal curvature and the temporal torsion, respectively, and $d \tau=\sqrt{d \mathbf{t} . d \mathbf{t}}$ is the linear element of a temporal curve. If we only consider the motion of a particle in a plane, the equations given in Equation (13) reduce to

$$
\frac{d \mathbf{t}_{T}}{d \tau}=\kappa \mathbf{p}, \frac{d \mathbf{p}}{d \tau}=-\kappa \mathbf{t}_{T}
$$

By differentiation we obtain the following system of differential equations

$$
\frac{d^{2} \mathbf{t}_{T}}{d \tau^{2}}-\frac{d(\ln \kappa)}{d \tau} \frac{d \mathbf{t}_{T}}{d \tau}+\kappa^{2} \mathbf{t}_{T}=0
$$

$$
\frac{d^{2} \mathbf{p}}{d \tau^{2}}-\frac{d(\ln \kappa)}{d \tau} \frac{d \mathbf{p}}{d \tau}+\kappa^{2} \mathbf{p}=0 .
$$

If the temporal curvature $\kappa(\tau)$ is assumed to vary slowly along the curve $\mathbf{t}(\tau)$, so that the condition $d(\ln \kappa) / d \tau=0$ can be imposed, then $\mathbf{t}_{T}(\tau)$ and $\mathbf{p}(\tau)$ may be regarded as being oscillating with a temporal period $T$, whose relationship with the temporal curvature $\kappa$ is found from the differential equations given in Equation (15) or (16) as

$$
\kappa=\frac{2 \pi}{T}
$$

This result shows that the temporal curvature $\kappa$ is actually the angular frequency $\omega$. In principle, the structure of the three-dimensional spatial manifold and the three-dimensional temporal manifold are identical, because, without matter, both of them are just a three-dimensional Euclidean continuum. In order to incorporate this elementary differential geometry into quantum mechanics, we identify the angular frequency defined in Equation (17) with the angular frequency in Planck's quantum of energy $E=\hbar \omega$ of a particle. With this assumption, the energy of the particle and the curvature $\kappa$ are related through the relation

$$
E=\hbar \kappa \text {. }
$$

As in the case of Bohr's quantisation of angular momentum, the quantisation of energy can be obtained from the relation given by Equation (18) using the principle of least action for the temporal manifold and it can be said that the quantisation of energy is a manifestation of rotation, or oscillation, in the two-dimensional temporal manifold.

In fact, the Feynman's method of sum over random paths can be extended to higher-dimensional spaces to formulate physical theories in which the transition amplitude between states of a quantum mechanical system is the sum over random hypersurfaces. This generalisation of the path integral method in quantum mechanics has been developed and applied to other areas of physics, such as condensed matter physics, quantum field theories and quantum gravity theories, mainly for the purpose of field quantisation. For example, although there are conceptual difficulties, the path integral approach to quantum gravity is carried out by considering a sum over all field configurations, determined by a metric $g_{\mu \nu}$ and matter field $\phi_{\mu}$, which are consistent with the three-geometries at the boundaries of the space-time [18]. On the other hand, string theories can be formulated in terms of a sum over random surfaces [19]. In this case the surface integral method can be used in the quantisation procedure, where the surface action of the form $S=-(1 / 2 \pi) \int d^{2} \sigma \sqrt{h} h^{\mu v} \partial_{\mu} X^{\alpha} \partial_{v} X^{\alpha}$ is used for bosonic strings, and $S=-(1 / 2 \pi) \int d^{2} \sigma\left(\sqrt{h} h^{\mu v} \partial_{\mu} X^{\alpha} \partial_{v} X^{\alpha}-i \psi^{\mu} \gamma^{\alpha} \partial_{\alpha} \psi_{\mu}\right)$ for fermionic strings. In these action integrals, the spatial coordinates $\sigma^{i}(i=1,2)$ describe a two-dimensional world sheet, the quantities $\gamma^{\mu}$ represent 2-dimensional Dirac matrices, the quantities $X^{\mu}(\sigma)$ are mappings from the world manifold into the physical space-time, and $h^{\mu \nu}$ represents the geometry of the 2-dimensional manifold. 
These surface actions are a generalisation of the familiar action integral for a point particle, $S=-m \int d s$, where the invariant interval is defined by the relation $d s^{2}=$ $g_{\mu \nu} d x^{\mu} d x^{\nu}$ [20]. In the following, however, we focus attention on the general idea of a sum over random surfaces. This formulation is based on surface integral methods by generalising the differential formulation as discussed for the Bohr's model of a hydrogen-like atom. Consider a surface in $\mathrm{R}^{3}$ defined by the relation $x^{3}=$ $f\left(x^{1}, x^{2}\right)$. The Gaussian curvature $K$ is given by the relation $K=\left(f_{11} f_{22}-\left(f_{12}\right)^{2}\right) /\left(1+f_{1}^{2}+f_{2}^{2}\right)^{2}$, where $f_{\mu}=\partial f / \partial x^{\mu}$ and $f_{\mu \nu}=\partial^{2} f / \partial x^{\mu} \partial x^{\nu}$ [14]. Let $P$ be a three-dimensional physical quantity which plays the role of the momentum $p$ in the two-dimensional space action integral. The quantity $P$ can be identified with the surface density of a physical quantity, such as charge. Since the momentum $p$ is proportional to the curvature $\kappa$, which determines the planar path of a particle, it is seen that in the three-dimensional space the quantity $P$ should be proportional to the Gaussian curvature $K$, which is used to characterise a surface. If we consider a surface action integral of the form $S=\int P d A=\int(q / 2 \pi) K d A$, where $q$ is a universal constant, which plays the role of Planck's constant, then we have

$$
S=\frac{q}{2 \pi} \int \frac{f_{11} f_{22}-\left(f_{12}\right)^{2}}{\left(1+f_{1}^{2}+f_{2}^{2}\right)^{3 / 2}} d x^{1} d x^{2} .
$$

According to the calculus of variations, similar to the case of path integral, to extremise the action integral $S=\int L\left(f, f_{\mu}, f_{\mu \nu}, x^{\mu}\right) d x^{1} d x^{2}$, the functional $L\left(f, f_{\mu}, f_{\mu \nu}, x^{\mu}\right)$ must satisfy the differential equations [16]

$$
\frac{\partial L}{\partial f}-\frac{\partial}{\partial x^{\mu}} \frac{\partial L}{\partial f_{\mu}}+\frac{\partial^{2}}{\partial x^{\mu} \partial x^{\nu}} \frac{\partial L}{\partial f_{\mu v}}=0 .
$$

Also as in the case of path integral, it is straightforward to verify that with the functional of the form $L=(q / 2 \pi)\left(f_{11} f_{22}-\left(f_{12}\right)^{2}\right) /\left(1+f_{1}^{2}+f_{2}^{2}\right)^{3 / 2}$ the differential equations given by Equation (20) are satisfied by any surface. Hence, we can generalise Feynman's postulate to formulate a quantum theory in which the transition amplitude between states of a quantum mechanical system is a sum over random surfaces, provided the functional $P$ in the action integral $S=\int P d A$ is taken to be proportional to the Gaussian curvature $K$ of a surface. Consider a closed surface and assume that we have many such different surfaces which are described by the higher dimensional homotopy groups. As in the case of the fundamental homotopy group of paths, we choose from among the homotopy class a representative spherical surface, in which case we can write

$$
\oint P d A=\frac{q}{4 \pi} \oint d \Omega,
$$

where $d \Omega$ is an element of solid angle. Since $\oint d \Omega$ depends on the homotopy class of the sphere that it represents, we have $\oint d \Omega=4 \pi n$, where $n$ is the topological winding number of the higher dimensional homotopy group. From this result we obtain a generalised Bohr quantum condition

$$
\oint P d A=n q .
$$

From the result obtained in Equation (22), as in the case of Bohr's theory of quantum mechanics, we may consider a quantum process in which a physical entity transits from one surface to another with some radiation-like quantum created in the process. Since this kind of physical process can be considered as a transition from one homotopy class to another, the radiation-like quantum may be the result of a change of the topological structure of the physical system, and so it can be regarded as a topological effect. Furthermore, it is interesting to note that the action integral $(q / 4 \pi) \oint K d A$ is identical to Gauss's law in electrodynamics [21]. In this case the constant $q$ can be identified with the charge of a particle, which represents the topological structure of a physical system and the charge of a physical system must exist in multiples of $q$. Hence, the charge of a physical system may depend on the topological structure of the system and is classified by the homotopy group of closed surfaces. This result may shed some light on why charge is quantised even in classical physics. As a further remark, we want to mention here that in differential geometry, the Gaussian $K$ is related to the Ricci scalar curvature $\mathrm{R}$ by the relation $\mathrm{R}=2 \mathrm{~K}$. And it has been shown that the Ricci scalar curvature can be identified with the potential of a physical system, therefore our assumption of the existence of a relationship between the Gaussian curvature and the surface density of a physical quantity can be justified [11]. Our discussions for random surfaces can also be generalised to a sum over random hypersurfaces of the form $x_{n+1}=f\left(x_{1}, x_{2}, \ldots, x_{n}\right)$ in an $(n+1)$-dimensional Euclidean space. A generalised action integral is assumed to take the form

$$
S=\int q_{n} K_{n} d A_{n},
$$

where $q_{n}$ is a universal constant, which may be identified with some physical quantity, depending on the dimension of space, and $K_{n}$ is the generalised Gaussian curvature defined as the product of the principal curvatures $K_{n}=$ $k_{1} k_{2} \ldots k_{n}$. In terms of the Riemannian curvature tensor, the generalised Gaussian curvature can be written as

$$
K_{n}=\frac{\epsilon^{\mu_{1} \ldots \mu_{n}} \epsilon^{v_{1} \ldots v_{n}}}{2^{\frac{n}{2}} n ! \operatorname{det}\left(g_{\mu v}\right)} R_{\mu_{1} \mu_{2} v_{1} v_{2}} \ldots R_{\mu_{n-1} \mu_{n} v_{n-1} v_{n}}
$$

where $\mu_{i}, v_{i}=1,2, \ldots n$ and $g_{\mu \nu}$ and $R_{\mu_{i} \mu_{i+1} v_{i} v_{i+1}}$ are the metric tensor and the curvature tensor, respectively, of the hyper-surface. From the theory of differential geometry in higher dimensions, we have the generalised Gauss-Bonnet theorem for the case when $n$ is even [22]

$$
\int K_{n} d A_{n}=\frac{1}{2} S^{n} \chi
$$

where $S^{n}$ is the volume of an $n$-sphere and $\chi$ is the Euler characteristic. Hence, to be consistent with this result the variational differential equation obtained from the action integral given by Equation (23) must be satisfied by any hypersurface, since the Euler characteristic is a topological invariant whose value depends only on the homotopy class, and not on the choice of an individual hypersurface. In this case we have the generalised Bohr quantum condition 


$$
\oint P_{n} d A_{n}=n q_{n}
$$

where the universal constant $q_{n}$ connects the physical quantity $P_{n}$ with the geometrical object $K_{n}$. In higher-dimensional spaces, however, we do not have a guiding relation, such as de Broglie's relation and Gauss's law to identify the quantity $q_{n}$. Nonetheless, this result also suggests that it may be possible to discuss a formalisation of the physics that involves manifolds of arbitrary dimension.

\section{Principle of Least Action in General Relativity}

As a further examination, we will show in this section that Feynman's postulate of path integral formulation of quantum mechanics, which can be verified by the principle of least action as discussed above, may also be applied to the field equations of Einstein's theory of general relativity. The field equations of general relativity are written in the form

$$
R_{\mu v}-\frac{1}{2} g_{\mu v} R+\Lambda g_{\mu v}=\kappa T_{\mu v}
$$

where $T_{\mu \nu}$ is the energy-momentum tensor, $g_{\mu \nu}$ is the metric tensor, $R_{\mu \nu}$ is the Ricci curvature tensor, $R$ is the scalar curvature and $\Lambda$ is the cosmological constant [23]. It is shown that Equation (27) can be derived through the principle of least action $\delta S=0$, where the action $S$ is defined as

$$
S=\int\left(\frac{1}{2 \kappa}(R-2 \Lambda)+\mathcal{L}_{M}\right) \sqrt{-g} d^{4} x
$$

where $\mathcal{L}_{M}$ characterises matter fields [24]. In 1917, Einstein introduced the cosmological constant $\Lambda$ as an addition to his original field equations of general relativity to retain the accepted view at the time that the universe is static. The reason for the addition is that if all matter attracts each other than a static universe would not be able to remain static. The attractive gravity would cause the universe to collapse. Because the original field equations of general relativity contain only attractive forms of gravity, a repulsive term is required. Einstein started his cosmological considerations by modifying Poisson's equation $\nabla^{2} \phi=4 \pi K \rho$ with a repulsive term $\lambda \phi$ to form the required equation $\nabla^{2} \phi-\lambda \phi=4 \pi \kappa \rho$, where $\lambda$ denotes a universal constant [23]. The modification of Poisson's equation results in the solution $\phi=-(4 \pi \kappa / \lambda) \rho_{0}$ if matter was distributed uniformly through space with the density $\rho_{0}$. With these considerations, Einstein also noted that in order to maintain the general covariance of the field equations, the repulsive term that would be added to his field equations must be of the form $\Lambda g_{\mu \nu}$. However, it is observed that the requirements of repulsion and covariance can be acquired by the energy-momentum tensor because if $\Lambda$ is considered to be some form of matter then it should be able to produce some form of energy, in particular, a potential energy. The fact that the term $\Lambda g_{\mu \nu}$ can be replaced by an energy-momentum tensor can be demonstrated for the case when $n=2$ as follows. It is shown that when $n=2$ we have the identity
$R_{\mu \nu}-\frac{1}{2} g_{\mu \nu} R \equiv 0$, and in this case the field equations given by Equation (27) reduces to

$$
T_{\mu v}=\left(\frac{\Lambda}{\kappa}\right) g_{\mu v} .
$$

It is indicated from Equation (29) that a physical entity can be directly identified with a mathematical object. This interesting feature can be seen as an underlying principle for quantum physics. As long as the energy-momentum tensor $T_{\mu \nu}$ is directly identified with the metric tensor $g_{\mu \nu}$ through the relationship given by Equation (29) then for the case when $n=2$ we have $R_{\mu \nu}-\frac{1}{2} g_{\mu \nu} R \equiv 0$, therefore the resulting equations from the principle of least action $R_{\mu v}-\frac{1}{2} g_{\mu v} R+\Lambda g_{\mu v}=\kappa T_{\mu v}$ are satisfied by any metric tensor $g_{\mu \nu}$.

\section{Conclusion}

In this work we have analysed the principle of least action that can be applied to various domains of physics, namely the classical mechanics, the quantum mechanics and the general relativity. We showed that the principle of least action in the traditional formulation not only can be applied to determine a unique path of a particle in classical mechanics but also can be applied to determine the quantum dynamics of a quantum particle with the Feynman assumption that the quantum particle can take any path to move in the spacetime continuum. These results lead to the possibility to speculate that the mathematical formulation of physical laws is not only a convenient mathematical method but there may be intrinsic relationships between physical entities and the mathematical objects. And the ultimate question that arises from such speculation is whether it is possible to formulate physics purely in terms mathematical objects by identifying them with physical entities.

\section{Acknowledgements}

I would like to thank the referees for their constructive comments and suggestions for the improvement of the paper. I would also like to thank the administration of SciEP for their advices at various stages of the preparation for the publication of this work.

\section{References}

[1] S. Hojman, Problem of the identical vanishing of Euler-Lagrange derivatives in field theory, Phys. Rev. D., 27, 451-453 (1983).

[2] S. Hojman, Symmetries of Lagrangians and of their equations of motion, J. Phys. A: Math. Gen., 17, 2399-2412 (1984).

[3] I. Yu. Krivsky and V. M. Simulik, Noether analysis of zilch conservation laws and their generalisation for the electromagnetic field, Theor. Math. Phys. 80, 864-874 (1989).

[4] I. Yu. Krivsky and V. M. Simulik, Noether analysis of zilch conservation laws and their generalisation for the electromagnetic field, Theor. Math. Phys. 80, 912-921 (1989). 
[5] W. I. Fushchych, I. Yu. Krivsky and V. M. Simulik, On vector and pseudo-vector Lagrangians for electromagnetic field, Nuovo Cim B. 103, 423-429 (1989).

[6] R. R. Lompay and A. N. Petrov, Covariant differential identities and conservation laws in metric-torsion theories of gravitation, $\mathrm{J}$. Math. Phys. 54, 1-30 (2013).

[7] N. Bohr, Phil. Mag. 26, 1 (1913).

[8] H. Goldstein, Classical Mechanics (Addison-Wesley Inc., Sydney, 1980).

[9] R. P. Feynman, Rev. Mod. Phys. 20, 367 (1948).

[10] Vu B Ho, Geometrical and Topological Methods in Classical and Quantum Physics (PhD thesis, Monash University, Australia, 1996).

[11] Vu B Ho, Spacetime Structures of Quantum Particles (Preprint, ResearchGate, 2017), viXra 1708.0192v1.

[12] W. Yourgrau and S. Mandelstam, Variational Principles in Dynamics and Quantum Theory (Dover Publications, New York, 1979).

[13] I. M. Singer and J. A. Thorpe, Lectures Notes on Elementary Topology and Geometry (Scott,Foresman \& Co., MIT, 1967).

[14] E. Kreyszig, Introduction to Differential Geometry and Riemannian Geometry (University of Toronto Press, 1975).
[15] A. Prastaro, Geometry of PDEs and Mechanics (World Scientific, Singapore, 1996).

[16] C. Lanczos, The Variational Principles of Mechanics (Dover Publications, New York, 1970).

[17] Vu B Ho, A Temporal Dynamics: A Generalised Newtonian and Wave Mechanics (Pre-print, ResearchGate, 2016), viXra $1708.0198 \mathrm{v} 1$.

[18] S. W. Hawking, General Relativity, An Einstein Centenary Survey, edited by S. W. Hawking and W. Israel (Cambridge University Press, 1979).

[19] A.M. Polyakov, Phys. Lett. B, 103, 207 (1981).

[20] M. B. Green, J. H. Schwarz and E. Witten, Superstring Theory (Cambridge University Press, Melbourne, 1988)

[21] J. D. Jackson, Classical Electrodynamics (John Wiley \& Sons, New York, 1975)

[22] M. Spivak, A Comprehensive Introduction to Differential Geometry (Publish or Perish, Berkerley, 1979)

[23] A. Einstein, The Principle of Relativity (Dover Publications, New York, 1952).

[24] R. M. Wald, General Relativity (The University of Chicago Press, London, 1984). 